\title{
MINIMALISMO, ASPECTO Y PREDICADOS SECUNDARIOS
}

\author{
Ángel Jiménez Fernández
}

\begin{abstract}
In the generative literature there have been many proposals concerning secondary predicates which are based on the syntax of these constructions. Linguists have paid attention to mainly three issues: 1) the difference between subject and object oriented predicates, 2) the generation of secondary predicates in the VP or IP and the c-command relation which is established between the predicate and the subject which it refers to, and 3) the clausal status of these predicates. These questions are important, but they do not explain the (non)occurrence of seconday predicates in certain syntactic environments.
\end{abstract}

The proposal that I will present in this work is that lexical aspect imposes some constraints on the legitimation of these predicates. The starting hypothesis will be that only predicates that denote a temporary state or a state subject to change will receive grammatical licence. In a minimalist syntax, this aspectual restriction will be accounted for by proposing an aspectual phrase with a [+ perfective] feature. The aspectual feature of the predicate will be checked against ASP. If both features coincide the derivation will converge; otherwise it will crash.

\section{INTRODUCCIÓN}

En la literatura generativista ha habido una preocupación exacerbada por la sintaxis de los predicados secundarios, como se puede apreciar en los estudios realizados por Williams (1980), Rothstein (1983), Demonte (1989), Roberts (1988), McNulty (1988), Mallén (1991) y Aarts (1992). Fundamentalmente tres cuestiones han suscitado el interés de estos autores por los predicados secundarios: 1) la diferencia entre predicados orientados al sujeto y predicados orientados al objeto, 2) su origen dentro del SV o dentro del SFlex y la relación de mando-c entre el predicado y el sujeto al que se refieren, y 3) la naturaleza de cláusula de dichos predicados. Obviamente estas cuestiones son importantes, pero a mi entender no explican la habilitación de dichos predicados de una manera sistemática en ciertos contextos sintácticos.

El trabajo que presentaré aquí trata sobre las condiciones aspectuales que deben cumplir los predicados secundarios para recibir licencia gramatical. Se entenderá el aspecto léxico como una propiedad asociada a los predicados asignándoles un rasgo [- perfectivo] si denotan un estado permanente o un rasgo [+ perfectivo] si expresan un estado transitorio o 
alcanzado. Sostendré que sólo aquellos predicados que contengan el valor aspectual [+ perfectivo] pueden funcionar como predicados secundarios, tal come se ve en (1) y (2): ${ }^{1}$

(1) a. John chew the carrots raw.

b. *John chew the carrots huge.

(2) a. John left naked.

b. *Jonh left intelligent.

Siguiendo a Demonte (1991), Hernanz (1988) y Bosque (1990), aunque apartándome de éstos en la formulación de la propuesta concreta, postularé que la distribución de los predicados secundarios está constreñida por condiciones de índole semántica. Más específicamente, defenderé que el aspecto léxico con que cada predicado está marcado en el Lexicón es el encargado de habilitar una construcción predicativa.

Independientemente de si el predicado secundario es descriptivo o resultativo e independientemente de si se predica del sujeto u objeto matrices, dicho predicado debe estar marcado inherentemente como [+ perfectivo]. En otras palabras, debe hacer referencia a una propiedad denotadora de un cambio de estado. Si, por el contrario, el predicado secundario contiene el rasgo [- perfectivo] la oración será agramatical. Éste es el contraste que se da en (1-2).

La hipótesis que quiero formular es que estas constricciones semántico-aspectuales se proyectan en la sintaxis mediante un sintagma aspectual (SAsp) que contiene un núcleo nulo -en el sentido de Tenny (1987) y Cinque (1999)- cuyo rasgo ha de ser [+ perfectivo]. Dentro del marco minimalista de Chomsky (1995), este SAsp tendrá por complemento al SA del predicado secundario, cuyo rasgo aspectual se cotejará con el de ASP. Si dichos rasgos coinciden la derivación será convergente (gramatical), pero si se produce un desajuste de dichos rasgos la derivación se estrellará, dando lugar a una construcción agramatical. De esta manera se podrá dar cuenta del contraste que se producía en (1) y (2).

\footnotetext{
${ }^{1}$ La terminología aspectual que usaré en este trabajo parte de los estudios ya clásicos de Lyons (1968) y Comrie (1976), que clasifican el aspecto de los verbos como perfectivo o no-perfectivo. Siguiendo a Hernanz (1988) traslado estos términos a la información aspectual que pueden expresar el resto de categorías gramaticales. En gramática española existe una larga y notable tradición en el empleo de la dicotomía perfectivo/imperfectivo que data al menos de los estudios de Gili Gaya (1961) y que posteriormente adopta Luján (1981).

Paralelamente, el aspecto de los adjetivos se ha estudiado en términos de estatividad y dinamicidad (Lakoff 1966), aunque en estos trabajos se lleva a cabo una división semántica y no aspectual de los adjetivos, si bien ambas clases de divisiones coinciden en la progresión o no progresión del estado que expresa un determinado adjetivo. Básicamente lo que denomino perfectivo se corresponde con dinámico y lo que llamo no-perfectivo implica el mismo concepto que estativo. También quiero destacar la clasificación semántica de los verbos que realiza Chafe (1970), el cual distingue predicados de acción, proceso y estado perferctamente encajables en el presente trabajo, pues éste parte de la diferencia que establece Vendler (1967) entre estados, actividades, logros y realizaciones y que asumo plenamente. Esta división semántica restringe aún más la naturaleza de los predicados secundarios, objeto de mi estudio.
} 
La compatibilidad de rasgos aspectuales tendrá carácter no contrastivo en inglés y español, pues es un requisito que comparten ambas lenguas. Compárense los ejemplos (1-2) con (3):

(3) a. María llegó preocupada.

b. *María llegó atractiva.

c. María llegó más atractiva que nunca.

La diferencia gramatical en (3) se debe a las propiedades aspectuales del predicado secundario. Preocupada expresa un estado sujeto a cambio, mientras que atractiva puede indicar una propiedad permanente (3b) o temporal (3c). Sólo cuando el predicado secundario posee la valencia aspectual [+ perfectivo] puede habilitarse en estas construcciones. $^{2}$

Dichas estas notas introductorias, quiero avanzar cuál será la estructura que seguiré en mi trabajo. En un primer apartado dejaré claras las premisas sintácticas de las que parto. En segundo lugar, haré una clasificación aspectual de los adjetivos predicativos que condicionará la distribución de éstos como predicados secundarios. Finalmente, presentaré la derivación sintáctica de los predicados secundarios que se puede proponer dentro del marco del Programa Minimalista de Chomsky (1995).

\section{UNA APROXIMACIÓN SINTÁCTICA A LOS PREDICADOS SECUNDARIOS}

Para tener una visión global de la problemática que han creado los predicados secundarios (PPSS) en la historia de la Gramática Generativa, he de aclarar que se han dado dos posturas divergentes con respecto a la definición del sujeto. Por un lado, la teoría predicativa defiende que el sujeto es un concepto relacional en tanto se define por su relación con un predicado; por otro lado, la teoría de cláusulas reducidas estima que el sujeto es un elemento estructural dentro de toda oración.

Esta confrontación de perspectivas está estrechamente relacionada con la controversia que han creado las cláusulas reducidas, sean éstas argumentales o adjuntas (es decir, predicados secundarios). En la teoría predicativa estas construcciones consisten en una relación entre un sujeto y un predicado sin que medie ningún vínculo de cláusula entre ellos; ésta sería la visión defendida por Williams (1980, 1983), Napoli (1989, 1993), Culicover y Wilkins (1986) y Schein $(1981,1995)$. Para los proponentes de la cláusula reducida ésta comporta una estructura oracional, entre los que cabe destacar a Chomsky (1981, 1986), Stowell (1983) y Aarts (1992). Será esta última la posición que adoptaré en

\footnotetext{
${ }^{2}$ Tal como me hace ver un revisor anónimo de Philologia Hispalensis, las cuestiones pragmáticas hacen que la mayoría de los adjetivos puedan cambiar su valor aspectual. Así, un adjetivo como alta posee el rasgo [perfectivo] por tratarse de una propiedad permanente. Sin embargo, el contexto puede hacer que este mismo adjetivo modifique su valencia aspectual para expresar un cambio de estado (Llegó de Italia más alta de lo que era cuando se fue). Éste es el mismo caso del adjetivo enfermizo, el cual denota normalmente un estado permanente aunque puede cambiar su valencia aspectual, en cuyo caso puede aparecer como PS (*María llegó enfermiza/María llegó más enfermiza que nunca).
} 
este trabajo. Por tanto, siguiendo el concepto de sujeto que se asume en el modelo de Principios y Parámetros, definiré el sujeto como cualquier sintagma determinante (SD) que esté inmediatamente dominado por la oración, cuya realización sintáctica sería SFlex. Así las cosas, dependiendo de la noción de sujeto que se adopte, las construcciones predicativas como the details patchy en (4a) tendrían distinto análisis en la teoría predicativa (4b), donde no media un nudo de cláusula entre el SD the details y el predicado patchy, y en la teoría chomskyana (4c) donde X simbolizaría el nudo oracional en que se materializa la relación predicativa existente entre the details y patchy.:

(4) a. Wright found the details patchy.

b. Wright found [SD the details] [sA patchy].

c. Wright found [x[sDthe details] [sApatchy]].

Otro problema que plantean las construcciones predicativas tanto en la gramática tradicional como en la teoría predicativa es que no distinguen entre lo que se conoce como cláusula reducida argumental y predicado secundario. Como ilustración, presento en (5) ambos tipos de construcciones, cuyo análisis sería idéntico desde esta perspectiva:

(5) a. $\mathrm{Sam}_{\mathrm{i}}$ ate the cauliflower nude $\mathrm{i}$.

b. Sam ate the cauliflower cooked $_{\mathrm{i}}$.

c. Sam thought the cauliflower cooked $_{\mathrm{i}}$.

Los subíndices muestran de qué elemento se predican los adjetivos nude o cooked. Lo importante sobre este análisis es que resulta imposible distinguir cuándo el verbo matriz selecciona el SD que le sigue a modo de objeto y cuándo la relación es entre $\mathrm{V}$ y toda la secuencia postverbal.

Frente a este análisis, asumo con Stowell (1983) que la cadena [SD SA] en (5c) forman una cláusula reducida argumental, mientras que los SSAA en (5a-b) formarían un predicado secundario, el cual sería totalmente opcional, nunca un elemento regido por el verbo principal. Por esta razón, estos predicados secundarios son omisibles, lo cual no afectará a la sintaxis del V matriz:

(6) a. Sam ate the cauliflower.

b. Sam ate the cauliflower.

c. *Sam thought the cauliflower.

La definición exacta de predicado secundario será la que nos proporcionan Culicover y Wilkins (1986:122) y Demonte (1989:1): aquella categoría sintagmática que no mantiene relación gramatical con el $\mathrm{V}$ matriz y que se predica de un argumento marcado temáticamente por otro núcleo léxico. En (5a) nude no tiene relación de selección con el V 
eat, el cual asigna el rol temático de AGENTE al sujeto Sam, del cual se predica el SA nude. En (5b) cooked se predica del objeto the cauliflower el cual recibe el rol semántico de TEMA o Afectado del V eat. Por último, en (5c) cooked no tiene relación directa con el V matriz think, pero se predica del SD the cauliflower, el cual no está marcado temáticamente por otro núcleo léxico que no sea el mismo predicado cooked. El V principal think en (5c) asigna el rol de TEMA, pero es toda la secuencia the cauliflower cooked y no el SD el que lo recibe (Carrier y Randall 1992:179). ${ }^{3}$

Bajo esta definición se engloban varios tipos de predicado secundario a los que ya he hecho referencia. En la línea de Mallén (1991) y Demonte (1989, 1991), cabe distinguir predicados orientados al sujeto -caso de (5a) - y predicados orientados al objeto -caso de (5b). Otros ejemplos son (7a) y (7b) respectivamente:

(7) a. John always buys the meat worried.

b. John always buys the meat fresh.

La propiedad de los predicados secundarios más controvertida es su estatus de cláusula. Brevemente voy a construir un argumento que demostrará, a mi juicio, que efectivamente las cláusulas reducidas (o CCRR) adjuntas constituyen unidades oracionales de algún tipo. Aarts (1992) presenta varias pruebas para defender la naturaleza oracional de las CCRR argumentales, aquellas que van con verbos como considerar, esperar, etc. Lo que trataré de hacer yo será extender una de estas pruebas a los PPSS. En concreto, me basaré en la interpolación de adverbiales oracionales, es decir, aquellos que se generan dominados por la oración, tales como probably o perhaps. Si es posible encontrar este tipo de adverbio modificando a un PS, esto constituirá una prueba ineludible de que dicho PS se proyecta en un nudo oracional. En (8) se ven los ejemplos pertinentes:

(8) a. She will arrive probably exhausted.

b. She will probably arrive exhausted.

Si se comparan estas dos oraciones se podrá ver claramente que probably modifica al evento denotado por el $\mathrm{V}$ matriz arrive en (8b), mientras que se refiere al estado expresado por exhausted en (8a). Esto se traducirá sintácticamente en la formación de una unidad oracional de la que pendan tanto el predicado como el adverbio. En consecuencia, se puede afirmar que existe un nudo de cláusula en los PPSS.

Estructuralmente, estos PPSS no pueden formar una cláusula directamente con el SD al que se refieren. Necesitan un elemento que recoja la referencia de dicho SD y que funcione, pues, como sujeto del predicado secundario. El candidato para desempeñar estas funciones, según Hornstein y Lightfoot (1987), Schein (1995), Stowell (1995), inter alios, es sin duda

\footnotetext{
${ }^{3}$ Véase Jiménez (1998), donde se hace una incursión detallada por la estructura temática de las CCRR.
} 
el pronombre anafórico nulo PRO. El análisis que reciben las oraciones (5a-b) lo represento en $(9 a)$ y $(9 b)$ respectivamente:

(9) a. $\mathrm{Sam}_{\mathrm{i}}\left[\mathrm{sFlex}\left[\mathrm{sv}\left[\mathrm{v}\right.\right.\right.$ ate the cauliflower]] [ ${ }_{\mathrm{CR}} \mathrm{PRO}_{\mathrm{i}}$ nude]].

b. Sam [sv[v'[vate] the cauliflower $\left.{ }_{i}\right]\left[{ }_{C R} P O_{i}\right.$ cooked]].

He de hacer notar que PRO puede tomar como antecedente tanto al sujeto como al objeto de la oración principal, siempre que éstos guarden una relación de mando-c (Mallén 1991:381). Así, se distingue entre predicados orientados al sujeto -(9a)- y predicados orientados al objeto - (9b). Como queda manifiesto en (9), he asumido con Demonte (1989:13) que los predicados orientados al sujeto se adjuntan a la INFL, mientras que los orientados al objeto estarían dentro del SV. ${ }^{4}$

Semánticamente, hay que distinguir aquellos PPSS que describen a su referente de las que implican algún tipo de resultado de la acción del predicado matriz. De ahí que Demonte (1989), Simpson (1983) y Carrier y Randall (1992) clasifiquen a los predicados secundarios como depictive y resultative. En términos más estrictos, los predicados secundarios pueden ser interpretados como descriptivos - predicados que caracterizan situaciones temporales- 0 resultativos -predicados que se refieren a estados finales. Mallén (1991:385) nos facilita los siguientes ejemplos extraídos del español; el primero sería un descriptivo y el segundo un resultativo:

(10) a. Benjamín toma el café caliente.

b. Benjamín pintó el marco azul. ${ }^{5}$

Adviértase que esta clasificación está estrechamente relacionada con la que realizan Quirk et al. (1985), los cuales distinguen entre current y resulting para describir a los atributos. La diferencia reside en que Quirk et al. aplican esta distinción a lo que consideran complemento del objeto o del sujeto, no a los predicados secundarios caliente y azul en las oraciones anteriores. Es más, el tipo de construcción que se está estudiando aquí no ha sido analizada detalladamente en las gramáticas descriptivas, pues en la mayoría de los casos no se encuentra una distinción clara entre los predicados secundarios de (10) y las CCRR

\footnotetext{
${ }^{4}$ Roberts (1988) y McNulty (1988) afirman que los predicados del sujeto se generan adjuntados a la categoría máxima del SV, mientras que los predicados del objeto estarían ubicados en una posición más interna al V. Si se tiene en cuenta la hipótesis de que el sujeto se genera dentro del SV tendría sentido sostener que tanto los predicados orientados al sujeto como los orientados al objeto se originan internos al SV. No voy a entrar en este debate, puesto que queda fuera del alcance del trabajo que aquí presento.

${ }^{5}$ El uso de adjetivos que denotan color como resultativos del verbo pintar parece estar sujeto en español a discrepancias entre hablantes en torno a la inserción de la partícula de, que una gran mayoría de nativos sienten obligatoria (Benjamín pintó el marco de azul). El ejemplo que aparece en el texto es el que recoge Mallén (1991), para quien la lectura de resultativo es perfectamente posible a pesar de la ausencia de la partícula de. Por otra parte, parece que en situaciones metafóricas sí se podría obtener una interpretación resultativa con estos adjetivos de color sin que necesiten estar precedidos por partícula alguna, tal como me sugiere un revisor anónimo de Philologia Hispalensis (Pintar el panorama negro).
} 
argumentales que subcategorizan verbos como consider, make, expect, etc. Es más, Quirk et al. (1985: 1196-1198) clasifican verbos como estos últimos y los que pueden opcionalmente ir acompañado de un predicativo como paint, polish, sell, freeze, etc. en el mismo modelo de complementación. A modo de ilustración, tómense las entradas léxicas de los verbos boil y consider en el Diccionario Sintáctico del Verbo Inglés de Garrudo (1991). En ambos casos, se expone exactamente el mismo patrón de complementación, tal como se aprecia en (11), con la salvedad de que en la entrada de BOIL 6 /OC/ se especifica que procede de BOIL 2 /O/ por complementación del objeto: ${ }^{6}$

$$
\text { (11) a. Boil an egg hard. } \quad \text { /OC/ }
$$

b. We consider you innocent. /OC/

Una importante excepción es Jespersen (1909-1949), el cual traza una distinción entre elementos obligatorios y elementos opcionales con respecto a ambos tipos de CR. En (11a) el predicado hard tendría una función adverbial, frente a (11b) donde el predicado innocent forma una unidad con el sujeto you y sería toda la secuencia you innocent el objeto de consider.

\section{EL PAPEL DISCRIMINATORIO DEL ASPECTO LÉXICO EN LOS PPSS}

El objetivo de esta sección será dar cuenta de los requisitos de selección sintácticosemántica entre un verbo matriz y una CR adverbial o PS, que nos ayude a discernir la categoría máxima hasta la que se expande esta última, guiados por los principios de economía y el carácter reduccionista del PM de Chomsky (1995).

La hipótesis de partida es que el Aspecto influye de manera decisiva en la derivación óptima de una cláusula. La información aspectual aparece en la entrada léxica de algunas piezas junto a su red temática. Conjuntamente conforman la información léxica que se proyecta en la sintaxis (Demonte 1991 y De Miguel 1992), hipótesis a la que me adhiero. Sin embargo, reelaboraré esta idea para explicar ciertos contrastes gramaticales que muestran los PPSS.

La propuesta concreta que quiero formular es que las CCRR adverbiales, independientemente de si su predicado es descriptivo o resultativo e independientemente de si está orientado al sujeto o al objeto, incluyen la categoría funcional ASP especificada con los rasgos [ \pm perfectivo], la cual legitima la proyección que toma por complemento.

De otra parte, sostendré que en la derivación de un PS el rasgo aspectual de éste asciende al núcleo ASP para cotejar y eliminar dicha valencia aspectual y así hacer que la construcción converja en la FL.

\footnotetext{
${ }^{6}$ Tal como apunta un revisor de la revista, los supuestos teóricos de los que se parte en Garrudo (1991) no son comparables con las teorías minimistas que aquí se están asumiendo. Aunque en las entradas léxicas que se proporcionan para consider y boil aparece una información sintáctico-semántica detallada que los distingue, me interesa señalar que ésta no se refleja en los patrones sintácticos que se les asigna.
} 


\subsection{Una historia aspectual}

En la literatura se suelen encontrar dos tratamientos distintos del aspecto: Comrie (1976), Lyons (1977), Rojo (1988) y De Miguel (1992) plantean un concepto unitario del aspecto léxico y del aspecto morfológico, frente a la división que realizan autores como Bache (1982). En mi propuesta no haré distingos, pues en ambos casos el aspecto se concibe como manera de acción. Sin embargo, me interesa dejar bien sentado que en lo que concierne a las cláusulas averbales es el aspecto léxico el término relevante.

Entenderé el aspecto léxico como la temporalidad interna que denota un predicado. En palabras de Demonte (1991:123), la temporalidad interna es la manera de ver la acción expresada por un predicado o simplemente el modo de acción, canalizado en el concepto de Aktionsart (Vendler 1967).

Principalmente este estudio pretende formalizar el tipo de relación existente entre el aspecto léxico de una pieza y su habilitación como predicado de una CR adverbial. El contenido aspectual de una entrada léxica se debe proyectar de alguna manera en la sintaxis, tal como he indicado ya, mediante la inserción de un SAsp en la derivación de una cláusula.

Existe también cierta paradoja con respecto a la división entre predicados perfectivos y predicados imperfectivos, que no está exenta de incoherencias y que querré esclarecer. La dicotomía [ \pm perfectivo] se suele tomar en relación al hecho de si el evento denotado por el predicado está delimitado o no (Tenny 1987), o si el evento se ve como permanente o transitorio (Demonte 1991 y Raposo y Uriagereka 1995). No obstante, no parece haber acuerdo entre los autores sobre si un predicado perfectivo es permanente, no delimitado y acabado (Bosque 1990 y Hernanz 1988), o si dicho predicado perfectivo es temporal, delimitado e inacabado (Demonte 1991 y De Miguel 1992).

Al igual que Bosque (1990), este trabajo se va a guiar por la etimología del término "perfectivo". Así, un predicado perfectivo será aquel que exprese un evento sujeto a cambio, que se puede completar, mientras que un predicado imperfectivo implica un evento constante, ya completado. En otras palabras, "perfectivo" significará en este trabajo "perfeccionable", delimitable, temporal, susceptible de cambio.

El rasgo [ \pm perfectivo] será en nuestro marco teórico un valor semántico como cualquier otro, aunque sujeto a comprobación y seleccionador de una categoría compatible con dicho rasgo. Téngase en cuenta que en el PM la comprobación de rasgos responde a la necesidad de eliminar rasgos morfológicos. Estoy usando el concepto de aspecto en su sentido léxico y morfológico. Por tanto, parece natural que los valores aspectuales conlleven una compatibilización semántica y una comprobación morfológica. 
Esta compatibilización ha recibido distintos enfoques dentro del generativismo. ${ }^{7}$ Voy ahora a hacer un breve repaso por estas aproximaciones, que comparten el fin último de integrar la información aspectual en el Léxico, de forma que se pueda delimitar el camino que vamos a tomar para conseguir esta integración léxico-aspectual. Ha habido tres posturas a la hora de abordar esta inclusión:

1. Pustejovsky (1988), Jackendoff (1987), Grimshaw (1988) y Grimshaw y Vikner (1989) sostienen que junto a la red temática de un predicado existe una estructura aspectual que explicaría una serie de diferencias sintácticas a partir de distintos tipos de eventos denotados por un predicado.

2. Higginbotham $(1985,1989)$ sugiere integrar el aspecto en la entrada léxica de cada predicado haciendo uso de un argumento oculto o argumento davidsoniano -Donald Davidson (1967) fue el pionero en este terreno-. Este argumento eventivo sería parte de la estructura temática del predicado, el cual recoge los distintos tipos de evento y sobre el que giraría el resto de los roles- $\theta$.

Esta propuesta es seguida por autores como De Miguel (1992), con respecto a los verbos, y Hernanz (1988), Bosque (1990) y Ojea (1999), con respecto a otras clases de predicado: adjetivo, preposición y sustantivo.

3. Por último, Tenny (1987) señala la existencia de un nivel aspectual que relaciona el Léxico y la sintaxis. En realidad, la estructura sintáctica incluiría la aspectual, puesto que las posiciones sintácticas son las que poseen inherentemente las propiedades aspectuales canalizadas en la Aktionsart. Para Tenny $(1987,1994)$ y Verkuyl (1989) el aspecto léxico vendría determinado no sólo por el verbo sino a veces también por la relación de éste con sus complementos. De ahí la diferencia entre el estado perfectivo de la construcción Pedro lee el periódico y el estado imperfectivo de Pedro lee. ${ }^{8}$

De estas tres opciones, tomaré la propuesta de Tenny (1987), en el sentido de que la información aspectual hace de intermediaria entre el Lexicón y la sintaxis. En el Lexicón los predicados -tanto verbos como adjetivos, sustantivos y preposiciones- vienen marcados inherentemente con los rasgos [ \pm perfectivo]. En la selección de un predicado para entrar en la numeración de una derivación influyen estos rasgos, de forma que a la hora de

\footnotetext{
${ }^{7}$ Los autores que se citan llevan a cabo una integración léxico-aspectual sólo en la entrada de predicados verbales. Sin embargo, serán pauta inevitable en la aproximación que haré a otros tipos de predicado: adjetivo, preposición y sustantivo.

${ }^{8}$ Obviamente estas tres opciones aspectuales no deben verse como excluyentes, sino más bien como tratamientos distintos del aspecto que pueden ser incluso complementarios. De hecho, tal como me hace ver Demonte (c.p.), el argumento eventivo de Higginbotham (1985) no es incompatible con la hipótesis composicional del aspecto que defienden Tenny (1987) y Verkuyl (1989). La selección de un argumento de evento por parte de ciertos predicados hace que exista una estructura de intervalos y la consiguiente composición del aspecto. Los estados, al no tener argumento eventivo (Higginbotham 1989:471), no poseerían estructura eventiva, si bien también es verdad que Higginbotham (1985:555) admite la posibilidad de que los estados también tendrían una posición eventiva. Debido a esta controversia y al hecho de que no usaré el argumento de evento, no voy a entrar con detalle en la interrelación de las distintas propuestas aspectuales.
} 
expandirse una CS en el SAsp el rasgo de perfectividad de su predicado ha de ser compatible con el mismo valor que contiene el núcleo ASP.

La diferencia que se está trazando entre predicados perfectivos e imperfectivos ha tenido un correlato en otros términos. Así, Kratzer (1988, 1995), Chung y McCloskey (1987) y Raposo y Uriagereka $(1990,1995)$ distinguen entre predicados individuos (Individual-Level predicates) y predicados de estadio o etapa (Stage-Level predicates). Los primeros reciben una interpretación donde la propiedad asignada por el predicado de la CS se ve como permanente, mientras que los segundos poseen un predicado que denota una propiedad transitoria. $^{9}$

\subsection{La habilitación de los PPSS}

Entramos seguidamente en el meollo del asunto. Lo que quiero mostrar aquí es que tanto en español como en inglés la distribución de un PS está condicionada por su valor aspectual. Más estrictamente, basándome en los análisis de Hernanz (1988), Bosque (1990) y Demonte (1991) defenderé que lo que da licencia gramatical a un PS es el hecho de que haya seleccionado del Lexicón el rasgo aspectual [+ perfectivo] junto con sus otros rasgos formales de concordancia, es decir, que exprese un estado transitorio y no un estado permanente.

Para corroborar la veracidad de esta afirmación empezaré con el análisis del comportamiento de los PPSS en español, que aparecen de (11) a (14):

(11) a. Los soldados llegaron cansados.

b. *Los soldados llegaron honestos.

(12) a. Salí de la fiesta enamorado.

b. *Salí de la fiesta enamoradizo.

(13) a. María compró el libro destrozado.

b. *María compró el libro interesante.

(14) a. Pedro pintó la pared verde. ${ }^{10}$

b. *Pedro pintó la pared enorme.

\footnotetext{
${ }^{9}$ Kuroda (1972) se refiere a estos dos tipos de predicado como categorical y thetic respectivamente. Esta distinción también es asumida por Raposo y Uriagereka (1995).

${ }^{10}$ De nuevo surgen discrepancias entre los hablantes de español sobre el uso del adejetivo verde como resultativo en este ejemplo tomado de Demonte (1991:118). Parece que el español europeo es más dado a incluir la partícula de para la lectura resultativa, mientras que el español de América prefiere la ausencia de la partícula, a pesar de que se podría confundir el adjetivo con un mero postmodificador del núcleo puerta en el SD la puerta verde.
} 
Antes de esbozar una solución a los contrastes presentados, es interesante advertir de dos cuestiones. En primer lugar, los ejemplos (13b) y (14b) marcados como agramaticales, serían perfectamente lícitos con una lectura atributiva del adjetivo. Si se entiende toda la secuencia el libro interesante y la pared enorme como objeto de sus respectivos verbos. La cuestión es que si se fuerza la lectura predicativa de dichos adjetivos el resultado no sería correcto y éste es el caso que se estudia aquí. En segundo lugar, los predicados orientados al sujeto son a veces fácilmente confundibles con adverbios de modo, de forma que un adjetivo como débil en (15) sería parafraseable como un predicado secundario en (16a) o como un adverbio de manera en (16b), tal como apunta Demonte (1999):

(15) La enfermera tosió débil.

(16) a. La enfermera tosió y estaba débil en el momento de toser.

b. La enfermera tosió de manera débil aunque ya estaba recuperada y se sentía fuerte.

Conviene tener claro que se da este tipo de ambigüedades, pero en este trabajo sólo me dedicaré a la lectura predicativa de estos elementos. ${ }^{11}$

En el paradigma que se presenta en los ejemplos (11-14) hay PPSS orientados al sujeto, como en (11) y (12). También se dan predicados orientados al objeto como en (13) y (14). Asimismo, cabe destacar que el PS en (13) sería descriptivo, frente al de (14) que tendría un carácter marcadamente resultativo. La cuestión es que a pesar de estas diferencias sintáctico-semánticas, hay un requisito que deben cumplir todos los adjetivos que funcionen como PS. La generalización es que deben expresar una propiedad temporal, un estado sujeto posiblemente a cambio, nunca un estado permanente. En otras palabras, un PS debe ser un predicado de etapa marcado léxicamente con el rasgo aspectual [+ perfectivo].

Observemos que los adjetivos cansado, enamorado, destrozado y amarilla comparten la propiedad de designar un estado transitorio. Uno no está de por vida cansado, por ejemplo. Por tanto, estos adjetivos estarían marcados como [ + perfectivo]. Existen paralelamente adjetivos como amarilla que pueden tener una lectura aspectual ambivalente, pues puede hacer referencia a ser amarilla o estar amarilla. Precisamente, la diferencia en español entre ser y estar es la clave gramatical para saber a ciencia cierta cuál es el rasgo aspectual de un predicado, tal como proponen Luján (1981) y Hernanz (1988).

Por otro lado, los adjetivos honesto, enamoradizo, interesante o enorme describen un estado permanente y por consiguiente, se les asignaría en el Lexicón el rasgo [- perfectivo]. Por esta razón no se les puede dar licencia gramatical como PS. En inglés se encuentran los

\footnotetext{
${ }^{11}$ También me interesa aclarar que los PPSS a veces se pueden confundir con adverbios adjetivales, es decir, aquellas formas reducidas de los adverbios que terminan en -mente. Así, en oraciones como El pretendiente habló claro de sus pretensiones, tomada de Demonte (1999), la pieza claro no se predica del sujeto El pretendiente, sino que modifica al evento denotado por el SV habló de sus pretensiones y evidentemente puede sustituirse por el adverbio claramente, lo cual prueba el carácter de adverbio de modo de claro en la oración objeto de estudio. Sobre estas cuestiones ver Kovacci (1999), Di Tullio (1999) y Rodríguez Ramalle (1999).
} 
mismos contrastes con respecto a la habilitación de los adjetivos como PS, generalización que avalan los ejemplos (17-20):

(17) a. John drives drunk.

b. *John drives intelligent.

(18) a. Mary looked at them terrified.

b. *Mary looked at them stupid.

(19) a. They ate the carrots raw.

b. *They ate the carrots huge.

(20) a. She swept the room clean.

b. *She swept the room big.

De nuevo el contraste que distingue los ejemplos en (a) y (b) es la valencia aspectual del predicado secundario, ya que las oraciones en (a) serían gramaticales porque denotan un estado temporal y por tanto, estarían marcadas como [+ perfectivo]. Sin embargo, los ejemplos en (b) no son correctos debido a que expresan una propiedad permanente del elemento del que se predican. Sin embargo, merece la pena que nos preguntemos qué hace que sólo los PPSS que contengan el rasgo [+ perfectivo] puedan aparecer como tales tanto en inglés como en español. La respuesta reside en la compatibilidad de los predicados de etapa con el verbo matriz.

Siguiendo líneas trazadas por Vendler (1967), Dowty (1979) y Demonte (1991), asumiré que los verbos pueden dividirse en cuatro grupos: ESTADOS, ACTIVIDADES, LOGROS y REALIZACIONES, dependiendo de si hay actividad y/o resultado en el evento denotado por el verbo. Aunque la cuestión es peliaguda y necesitaría de una atención mucho más detallada, para no complicar las cosas en exceso, supondré con Hernanz (1988:26) que sólo los verbos dinámicos, nunca los estativos, pueden coaparecer con un PS, puesto que aquéllos son los únicos que expresan algún evento de cambio. Asimismo, también se podrá defender que un adjetivo que sea [-perfectivo] no puede ser compatible con un verbo dinámico, produciéndose un desajuste de rasgos aspectuales que llevará la construcción a la no convergencia. Así se explica el contraste en (21):

(21) a. *María adora a Mozart entusiasmada.

b. María juega a la pelota entusiasmada.

\subsection{El aspecto léxico y la derivación sintáctica de los PPSS}

En este último apartado quiero presentar brevemente un esbozo de la derivación sintáctica que debe tener un PS para converger o recibir licencia gramatical. Se ha visto que 
el aspecto léxico juega un papel crucial en la distribución sintáctica de los PPSS. Como antes mencioné, asumiré que la información aspectual se proyecta en la sintaxis mediante un núcleo funcional ASP que contendrá los rasgos [+ perfectivo] o [- perfectivo], en consonancia con las propuestas de Bosque (1990), Ojea (1999) o Demonte (1991). Sin embargo, en el sentido minimalista de Chomsky $(1995,1998)$ este rasgo atraerá o identificará una categoría que contenga la misma valencia aspectual para que se pueda eliminar. Esta categoría será el predicado secundario. No obstante, defenderé que este cotejo tiene lugar en la FL. Por tanto, sólo se moverá el rasgo aspectual y no la categoría que lo contiene. ${ }^{12}$

De otra parte, el rasgo de la categoría funcional ASP en los PPSS vendría condicionado por el carácter de acción del verbo principal. En otras palabras, ASP actuaría a modo de filtro de los requisitos de selección del V matriz, ya que éste condicionará el rasgo aspectual específico que se elige cuando se selecciona ASP desde el Lexicón.

Adicionalmente, como plantean French (1992), Chomsky y Lasnik (1995) y Baltin (1995), una CR adjuntiva contiene un sujeto nulo PRO. Ajustando esta propuesta a la hipótesis aspectual que aquí se defiende, se obtienen las representaciones que siguen para los PPSS orientados al objeto y para los orientados al sujeto:

(22) a. They painted the house $\mathrm{iPRO}_{\mathrm{i}}$ ASP red].

b. They ${ }_{i}$ painted the house $\left[\mathrm{PRO}_{\mathrm{i}}\right.$ ASP undressed].

Chomsky (1995) manifiesta que un sujeto nulo debe comprobar un caso nulo o abstracto, frente a la creencia estándar de que PRO no comprueba caso (Chomsky 1981). En este caso, siguiendo líneas trazadas por Sportiche (1995), en (22a) y (22b) PRO se genera como especificador de los SSAA [PRO red] y [PRO undressed], respectivamente. De la posición interna al SA PRO asciende para satisfacer el PPE. Por esta razón, se coloca en el especificador del SAsp de la CR adjuntiva. La disimilitud entre CR adjuntiva y CR completiva puede establecerse ahora en términos del dominio local en el que la CR se encuentra (Chomsky 1995, Bobaljik 1995).

Una cláusula completiva está en el dominio local de un $\mathrm{V}$ matriz, mientras que una cláusula adjuntiva no queda inmersa en este dominio local, más bien se adjunta en algún punto del SV, puesto que tanto si el PS es orientado al sujeto como orientado al objeto, el requisito de mando-c se cumpliría.

Por otro lado, la CR adjuntiva no está dominada localmente por el V paint en (22a-b). Por tanto, una vez que PRO se halla en el SAsp subordinado, permanece ahí. En esta posición comprueba no sólo los rasgos del PPE, sino también los rasgos de caso abstracto.

\footnotetext{
${ }^{12}$ La principal diferencia entre mi propuesta y la de Bosque (1990), Hernanz (1988) u Ojea (1999) reside en el hecho de que dentro del espíritu reduccionista de PM no defenderé que los predicados dinámicos contienen un argumento eventivo frente a los predicados estativos que carecerían de éste. Más bien, intento adaptar las restricciones aspectuales de los PPSS al mecanismo de cotejo de rasgos, con lo cual se evita la complejidad del sistema de cómputo, objetivo deseable desde el punto de vista minimalista.
} 
Para acabar, me gustaría representar más detalladamente las derivaciones que aquí se proponen para los PPSS, de forma que queden claros los diferentes conceptos que aquí se han expuesto. Para ello, me basaré en las oraciones en (22), cuyas derivaciones parciales respectivas se pueden ver en (23):

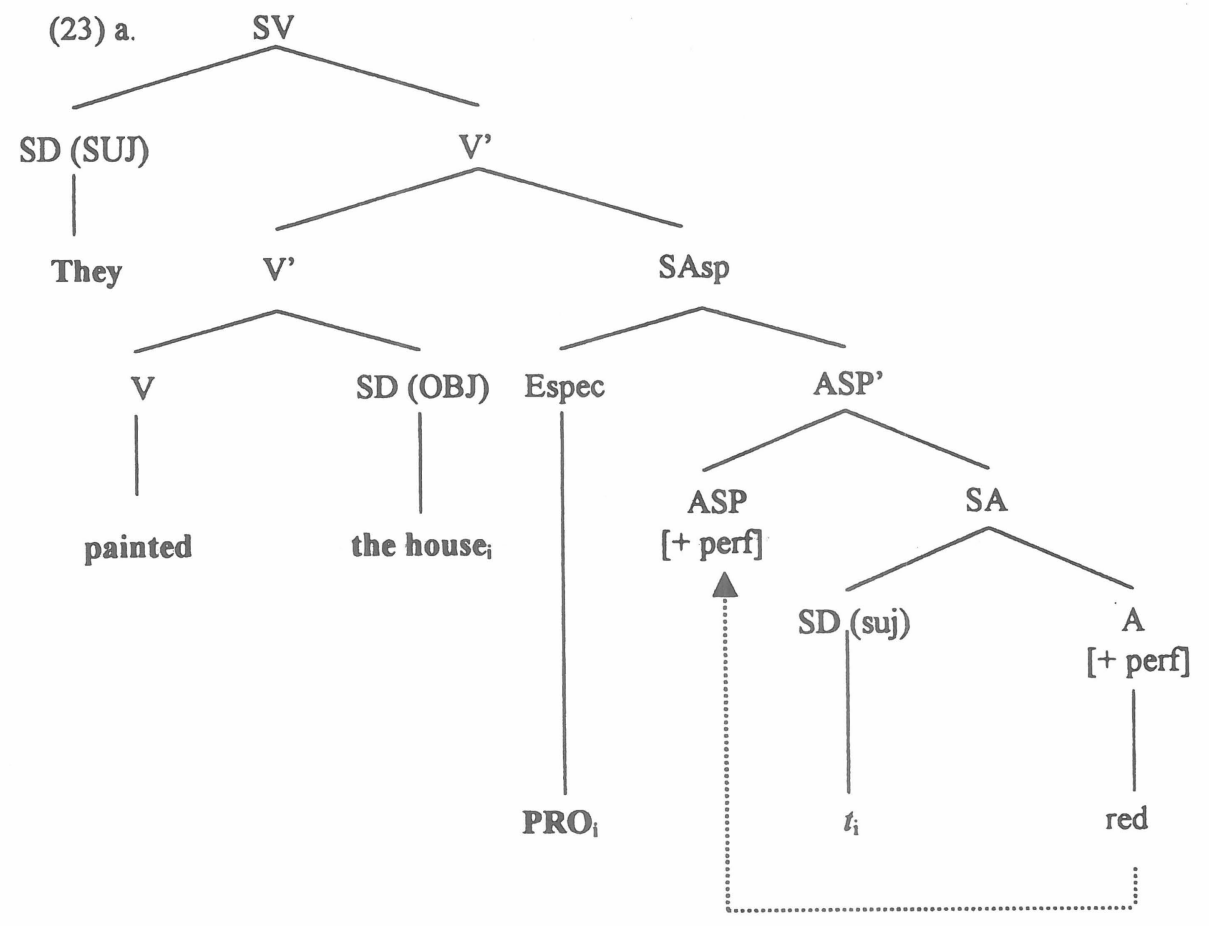

Tal como se puede observar, el requisito de localidad de Chomsky $(1995,1998)$ no se cumple en la derivación de los PPSS puesto que no son argumentos del V matriz, y por consiguiente, no estarían dominados localmente por éste. Otra propiedad de las derivaciones en (23) es que se distinguen perfectamente los predicados orientados al sujeto y los orientados al objeto en términos configuracionales: los primeros estarían adjuntados a la categoría intermedia V', los segundos se adjuntan a la categoría máxima SV. El requisito de mando-c se cumple en ambos casos, pues en (23a) the house manda-c a PRO y éste al predicado red. Por el contrario, en (23b) sería el sujeto matriz they antes de dejar su lugar de origen en el SV el que mandaría-c a PRO y éste al predicado undressed.

Con respecto a los rasgos aspectuales, la proyección de la categoría ASP es la que legitima la aparición de ambos PPSS red y undressed. Si entendemos el cotejo de rasgos como identidad de rasgos, podremos tener una base teórica para sostener que lo que hace converger las derivaciones en (23) es que en ambos casos se da identidad de los rasgos aspectuales de ASP y de los PPSS. Esta identidad no se comprobará hasta que la derivación esté en la FL, lo cual supone una derivación mucho más económica que si se produjera el 
desplazamiento explícito de la categoría que contiene el rasgo aspectual, es decir, el PS en cuestión.

(23) b.
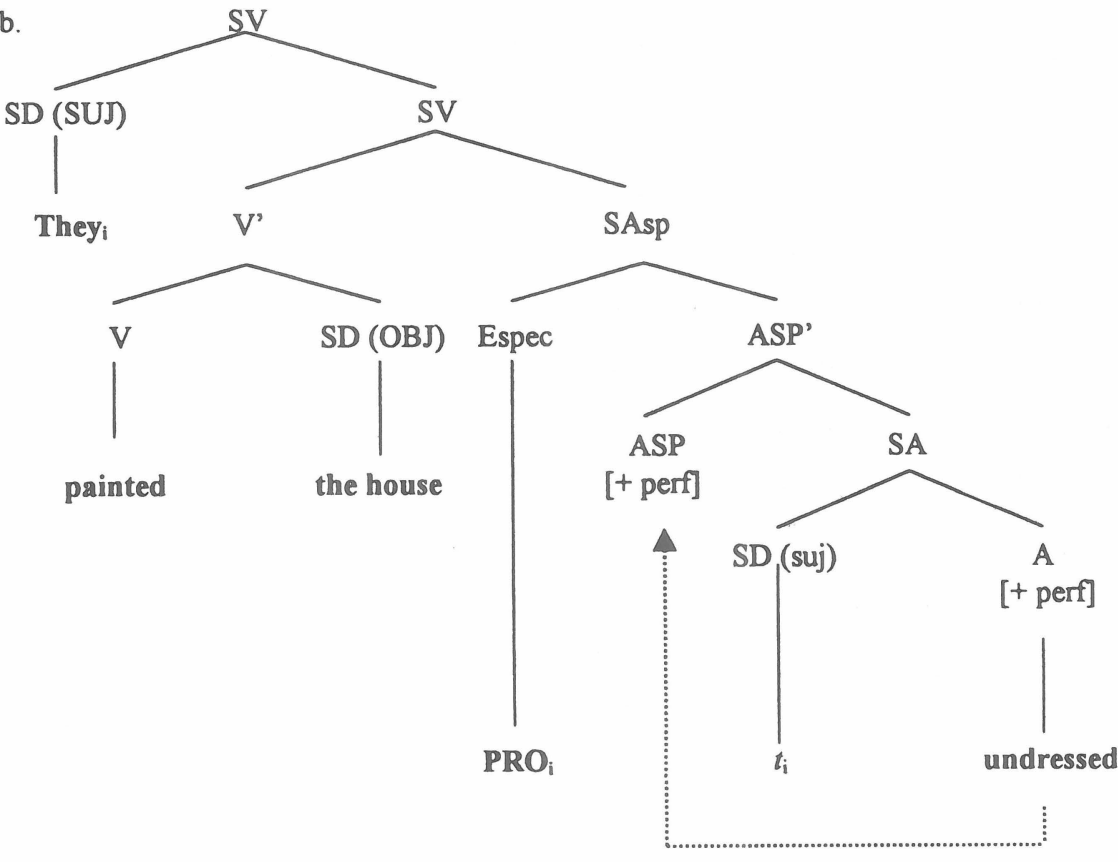

\section{CONCLUSIÓN}

En este trabajo he defendido que los predicados secundarios deben poseer un rasgo gramatical de aspecto que los capacita para actuar como tales. Concretamente, este rasgo de aspecto es [+ perfectivo], de forma que sea compatible con los requisitos de selección que impone el verbo matriz. Para dar cuenta de dichos requisitos, he postulado la existencia de la categoría funcional ASP, que servirá de filtro al tiempo que formará el dominio idóneo donde cotejar el rasgo [+ perfectivo] del PS una vez que la derivación se halla en la FL.

\section{REFERENCIAS}

Aarts, B. (1992) Small Clauses in English: The Non-Verbal Types. Berlin y New York: M. de Gruyter.

Bache, C. (1982) "Aspect and Aktionsart: Towards a Semantic Distinction", Journal of Linguistics 18, 57-72.

Baltin, M.R. (1982) “A Landing Site Theory of Movement Rules”, Linguistic Inquiry 13, 1 38. 
Baltin, M.R. (1995) "Floating Quantifiers, PRO, and Predication”, Linguistic Inquiry 26, 199-248.

Bobaljik, J.D. (1995) Morphosyntax: The Syntax of Verbal Inflection. Tesis Doctoral, MIT.

Bosque, I. (1990) "Sobre el Aspecto en los Adjetivos y en los Participios", en I. Bosque (ed.) Tiempo y Aspecto en Español. Madrid: Cátedra. 177-214.

Cardinaletti, A. y M.T. Guasti (eds.) (1995) Syntax and Semantics: Small Clauses Vol. 28. London y New York: Academic Press.

Carrier, J. y J. Randall (1992) "The Argument Structure and Syntactic Structure of Resultatives", Linguistic Inquiry 23, 173-234.

Chafe, W.L. (1970) Meaning and the Structure of Language. Chicago: University Press.

Chomsky, N. (1981) Lectures on Government and Binding. Dordrecht: Foris.

Chomsky, N. (1986) Barriers. Cambridge, MA: MIT Press.

Chomsky, N. (1995) The Minimalist Program. Cambridge, MA: MIT.

Chomsky, N. (1998) "Minimalist Inquiries: The Framework", MIT Occasional Papers in Linguistics 15. Cambridge, MA: MIT Press.

Chomsky, N. y H. Lasnik (1995) "The Theory of Principles and Parameters", en N. Chomsky (ed.) The Minimalist Program. 13-128.

Chung, S. y J. McCloskey (1987) "Government, Barriers, and Small Clauses in Modern Irish", Linguistic Inquiry 18, 173-237.

Cinque, G. (1999) Adverbs and Functional Heads. Oxford: O.U.P.

Comrie, B. (1976) Aspect: An Introduction to the Study of Verbal Aspect and Related Problem. Cambridge: C.U.P.

Culicover, P.W. y W. Wilkins (1986) "Control, PRO and the Projection Principle", Language 62, 120-153.

Davidson, D. (1967) "The Logical Form of Action Sentences", en Essays on Actions and Events. Oxford: Clarendon Press. 105-122.

Demonte, V. (1989) "Remarks on Secondary Predicates: C-Command, Extraction, and Reanalysis", The Linguistic Review 6, 1-39.

Demonte, V. (1991) Detrás de la Palabra: Estudios de Gramática del Español. Madrid: Alianza Editorial.

Demonte, V. (1999) "Minimalismo y Léxico: adjetivos y otras cuestiones", conferencia presentada en el seminario 50 años de Gramática Generativa: Propuestas para el siglo XXI. Universidad de Sevilla. 
Di Tullio, Á. (1999) “Adverbios con forma adjetival o adjetivos sin flexión”. Manuscrito inédito, Universidad Nacional de Comahue.

Dowty, D. (1979) World Meaning and Montague Grammar. Dordrecht: Reidel.

French, R. (1992) “Adjuncts, Small Clauses, and the $\theta$-Criterion”. Ponencia presentada en The Annual Meeting of the Linguistic Society of America, Philadelphia.

Garrudo, F. (1991) Diccionario Sintáctico del Verbo Inglés. vol. I, A-K. Barcelona: Ariel.

Gili Gaya, S. (1961) Curso superior de sintaxis española. Barcelona: Biblograf.

Grimshaw, J. (1988) “Adjuncts and Argument Structure”. Lexicon Project Working Papers 21, Center for Cognitive Science, MIT.

Grimshaw, J. y S. Vikner (1989) "Obligatory Adjuncts and the Structure of Events", conferencia presentada al Workshop on Lexical Structure of the Groningen Conference on Knowledge and Language.

Hernanz, M.L. (1988) "En Torno a la Sintaxis y la Semántica de los Complementos Predicativos en Español”, Estudi Generali 8, 7-29.

Higginbotham, J. (1985) “On Semantics”, Linguistic Inquiry 16, 547-594.

Higginbotham, J. (1989) "Elucidations of Meaning", Linguistics and Philosophy 12, 465 517.

Hornstein, N. y D.W. Lightfoot (1987) “Predication and PRO”, Language 63, 23-52.

Jackendoff, R.S. (1987) "The Status of Thematic Relations in Linguistic Theory", Linguistic Inquiry 18, 369-411.

Jespersen, O. (1909-1949) A Modern English Grammar on Historical Principles. London y Copenhagen: George Allan y Unwin y Munksgaard.

Jiménez, Á. (1998) Análisis de cláusula sintéticas subcategorizadas: modelo teóricodescriptivo. Tesis doctoral, Universidad de Sevilla.

Kovaccci, O. (1999) "El adverbio", en I. Bosque y V. Demonte (eds.) Gramática Descriptiva de la Lengua Española. Madrid: RAE-Espasa Calpe. 705-786.

Kratzer, A. (1988) Stage-Level and Individual Predicates. Manuscrito inédito, University of Massachussetts, Amherst.

Kratzer, A. (1995) "Stage-level and Individual-level Predicates", en G. Carlson y F. Pelletier (eds.) The Generic Book. Chicago: The University of Chicago Press. 176-223.

Kuroda, S.-Y. (1972) "The Categorical and the Thetic Judgment: Evidence from Japanese Syntax", Foundations of Language 9, 153-185.

Lakoff, G. (1966) "Stative Adjectives and verbs in English", Mathematical Linguistics and Automatic Translation, Report NSF-17. Cambridge, MA: The Computational Laboratory of Harvard University. 1-16. 
Luján, M. (1981) “The Spanish Copulas as Aspectual Indicators”, Lingua 54, 165-209.

Lyons, J. (1977) Semantics. Londres: C.U.P.

Mallén, E. (1991) “A Syntactic Analysis of Secondary Predication in Spanish”, Journal of Linguistics 27, 375-403.

McNulty, E. (1988) The Syntax of Adjunct Predicates. Tesis doctoral inédita, University of Connecticut.

Miguel, E. De (1992) El Aspecto en la Sintaxis del Español: Perfectividad e Impersonalidad. Madrid: Ediciones UAM.

Napoli, D.J. (1989) Predication Theory: A Case Study for Indexing Theory. Cambridge: CUP.

Napoli, D.J. (1993) Syntax: Theory and Problems. New York y Oxford: Oxford University Press.

Ojea, A. (1999) "On the Eventive Structure of Adjectives and Adverbs", ATLANTIS 20, 159-176.

Pustejovsky, J. (1988) "The Geometry of Events", en C. Tenny (ed.) Studies in Generative Approaches to Aspect. Lexicon Project Working Papers 24. Cambridge, MA: Center for Cognitive Sciences. 19-39.

Quirk, R., S. Greenbaum, G. Leech y J. Svartvik (1985) A Comprehensive Grammar of the English Language. London y New York: Longman

Raposo, E. y J. Uriagereka (1995) "Two Types of Small Clauses (Toward a Syntax of Theme/Rheme Relations", en A. Cardinaletti y M.T. Guasti (eds.), Syntax and Semantics: Small Clauses. 179-276.

Riemsdijk, H. van y E.S. Williams (1986) Introduction to the Theory of Grammar. Cambridge, MA: MIT Press.

Roberts, I. (1988) "Predicative APs”, Linguistic Inquiry 19, 703-710.

Rodríguez Ramalle, T. (1999) Algunos aspectos de la sintaxis y semántica de los adverbios y de ciertas construcciones adverbiales. Tesis doctoral inédita, Universidad Autónoma de Madrid.

Rojo, G. (1988) “Temporalidad y Aspecto en el Verbo Español”, LEA X, 195-216.

Rothstein, S. (1983) The Syntactic Forms of Predication. Tesis Doctoral inédita, MIT.

Schein, B. (1981) "The S'-Deletion Parameter and Small Clauses", GLOW Newsletter 6, 49-51.

Schein, B. (1995) "Small Clauses and Predication", en A. Cardinaletti y M.T. Guasti (eds.). 49-76. 
Simpson, J. (1983) "Resultatives", en Levin, Rapaport y Zaenen (eds.) Papers in Lexical Functional Grammar. IUCL.

Sportiche, D. (1995) "French Predicate Clitics and Clause Structure", en A. Cardinaletti y M.T. Guasti (eds.). 287-324.

Stowell, T. (1983) "Subjects across Categories", The Linguistic Review 2, 285-312.

Stowell, T. (1995) "Remarks on Clause Structure”, en A. Cardinaletti y M.T. Guasti (eds.), 271-286.

Tenny, C. (1987) Grammaticalizing Aspect and Affectedness. Tesis Doctoral inédita, MIT.

Tenny, C. (1994) Aspectual Roles and the Syntax-Semantics Interface. Kluwer Academic Publishers: Dordrecht

Vendler, Z. (1967) Linguistics in Philosophy. Ithaca, NY: Cornell University Press.

Verkuyl, H. (1989) “Aspectual Classes and Aspectual Composition”, Linguistics and Philosophy 12, 39-94.

Williams, E. (1980) "Predication”, Linguistic Inquiry 11, 203-238.

Williams, E. (1983) “Against Small Clauses”, Linguistic Inquiry 14, 287-308. 
